\title{
Adequate concentration of B cell leukemia/lymphoma 3 (Bcl3) is required for pluripotency and self-renewal of mouse embryonic stem cells via downregulation of Nanog transcription
}

\author{
Songhwa Kang ${ }^{1}$, Jisoo Yun ${ }^{1}$, Da Yeon Kim ${ }^{1}$, Seok Yun Jung ${ }^{1}$, Yeon Ju Kim ${ }^{1}$, Ji Hye Park ${ }^{1}$, Seung Taek Ji ${ }^{1}$, Woong Bi Jang ${ }^{1}$, \\ Jongseong $\mathrm{Ha}^{1}$, Jae Ho Kim ${ }^{2}$, Sang Hong Baek ${ }^{3, *}$ E Sang-Mo Kwon, ${ }^{1,2}$ \\ ${ }^{1}$ Laboratory for Vascular Medicine and Stem Cell Biology, Medical Research Institute, Department of Physiology, School of Medicine, Pusan \\ National University, Yangsan 50612, ${ }^{2}$ Research Institute of Convergence Biomedical Science and Technology, Pusan National University \\ Yangsan Hospital, Yangsan 50612, ${ }^{3}$ Laboratory of Cardiovascular Disease, Division of Cardiology, School of Medicine, The Catholic \\ University, Seoul 06591, Korea
}

B cell leukemia/lymphoma 3 (Bcl3) plays a pivotal role in immune homeostasis, cellular proliferation, and cell survival, as a co-activator or co-repressor of transcription of the NF-kB family. Recently, it was reported that $\mathrm{BCl} 3$ positively regulates pluripotency genes, including Oct4, in mouse embryonic stem cells (mESCs). However, the role of $\mathrm{Bcl} 3$ in the maintenance of pluripotency and self-renewal activity is not fully established. Here, we report the dynamic regulation of the proliferation, pluripotency, and self-renewal of mESCs by $\mathrm{Bcl} 3$ via an influence on Nanog transcriptional activity. Bcl3 expression is predominantly observed in immature mESCs, but significantly decreased during cell differentiation by LIF depletion and in mESC-derived EBs. Importantly, the knockdown of Bcl3 resulted in the loss of self-renewal ability and decreased cell proliferation. Similarly, the ectopic expression of $\mathrm{Bcl} 3$ also resulted in a significant reduction of proliferation, and the self-renewal of mESCs was demonstrated by alkaline phosphatase staining and clonogenic single cell-derived colony assay. We further examined that Bcl3-mediated regulation of Nanog transcriptional activity in mESCs, which indicated that Bcl3 acts as a transcriptional repressor of Nanog expression in mESCs. In conclusion, we demonstrated that a sufficient concentration of $\mathrm{Bcl} 3$ in mESCs plays a critical role in the maintenance of pluripotency and the self-renewal of $\mathrm{mESCs}$ via the regulation of Nanog transcriptional activity. [BMB Reports 2018; 51(2): 92-97]

${ }^{*}$ Corresponding authors. Sang-Mo Kwon, Tel: +82-51-510-8075; Fax: +82-51-510-8076, E-mail: smkwon323@pusan.ac.kr, smkwon323@ hotmail.com; Sang Hong Baek, Tel: +82-2-2258-6030; Fax: +822-591-3614; E-mail: whitesh@catholic.ac.kr

https://doi.org/10.5483/BMBRep.2018.51.2.219

Received 20 November 2017, Revised 20 December 2017, Accepted 9 January 2018

Keywords: Bcl3, Mouse embryonic stem cell, Nanog, Self-renewal

\section{INTRODUCTION}

Embryonic stem cells (ESCs) possess the abilities of selfrenewal and pluripotency, and can differentiate into three germ layers (1). The pluripotency of ESCs is maintained by core regulatory factors, including Oct4, Sox2, and Nanog (2). Nanog is a critical transcription factor for maintenance of ESCs and regulation of the transcriptional network in the undifferentiated state (3-5). Nanog is required to establish ESC ground state pluripotency and plays an essential role in early embryonic development; Nanog-null ESCs are prone to loss of self-renewal and experience multilineage differentiation (6). In ESCs, Nanog is constitutively expressed by core regulatory factors, such as Oct4, Sox2, and Esrrb, which bind to the promoter region of Nanog (7-9).

B cell leukemia/lymphoma $3(\mathrm{Bcl} 3)$ was found to be overexpressed in B-cell chronic lymphocytic leukemia (10). Furthermore, the overexpression of $\mathrm{Bcl} 3$ is associated with several cancers, such as classical Hodgkin's lymphoma, and solid tumors (11). Bcl3 is classified as a member of the $I \kappa B$ family, containing seven ankyrin repeat domains that interact with homodimers of NF-kB p50 or p52 $(12,13)$. Bcl3 has been reported to act as a co-activator of transcription that interacts with the mitogenic transcription factor AP-1 (14) and retinoid $\mathrm{X}$ receptor (15). Bcl3 is also a negative regulator of TORC3mediated transcription from the human T-cell leukemia virus type 1 LTR (16). Although $\mathrm{Bcl} 3$ appears to function as a transcription co-regulator in hematopoietic and solid tumors, its role in other cells, including ESCs, is largely unknown. It was recently reported that ectopic $\mathrm{Bcl} 3$ expression positively regulated mouse ESC (mESC) pluripotency and promoted Oct4 promoter activities, whereas silencing of $\mathrm{Bcl} 3$ downregulates the expression of self-renewal-related gene expression patterns and facilitates cell differentiation (17). However, the precise contribution of $\mathrm{Bcl} 3$ to the maintenance of pluripotency and self-renewal activity has not been clarified.

Here, we investigated whether the gain of function or loss of

ISSN: 1976-670X (electronic edition)

Copyright (c) 2018 by the The Korean Society for Biochemistry and Molecular Biology

(c) This is an open-access article distributed under the terms of the Creative Commons Attribution Non-Commercial License (http://creativecommons.org/licenses/by-nc/4.0) which permits unrestricted non-commercial use, distribution, and reproduction in any medium, provided the original work is properly cited. 
function of $\mathrm{Bcl} 3$ expression might influence the maintenance of pluripotency and self-renewal activity in mESCs. Unexpectedly, excessive or insufficient concentration resulted in a similar suppression of pluripotency, self-renewal activity, and cell proliferation of mESCs by acting as a negative regulator of Nanog transcription in mESCs, which suggested that a precise concentration of $\mathrm{Bcl} 3$ might be critical for the proliferation and self-renewal of $\mathrm{mESCs}$.

\section{RESULTS}

\section{Bcl3 is highly expressed in mouse embryonic stem cells}

To determine the role of $\mathrm{Bcl} 3$ in $\mathrm{mESCs}$, we first measured $\mathrm{Bcl} 3$ protein levels in mESCs (E14 cells) and mouse embryonic fibroblasts (MEF). As shown in Fig. 1A, protein levels of $\mathrm{Bcl} 3$ were significantly higher in E14 compared with MEF, which suggested that $\mathrm{BCl} 3$ was highly expressed in undifferentiated mESCs. To investigate the expression profile of $\mathrm{Bcl} 3$ at the differentiation stage, we performed LIF depletion or incubated cells with low concentrations of serum to form ESC-derived embryoid bodies. In LIF-deficient conditions, the expression of $\mathrm{BCl} 3$ was rapidly decreased in a time-dependent manner (Fig. 1B), which was also previously reported to occur in the Nanog protein level. Furthermore, compared with undifferentiated
A

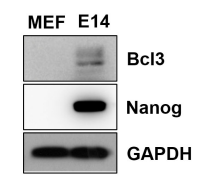

C

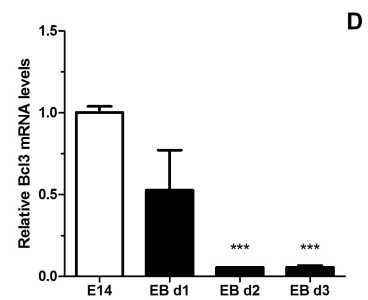

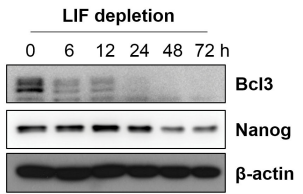

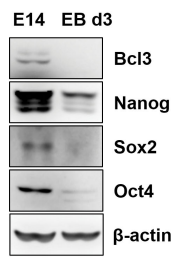

Fig. 1. Bcl3 is highly expressed in mouse embryonic stem cells. (A) Western blotting analysis of $\mathrm{Bcl} 3$ in mouse embryonic fibroblasts (MEF) and E14tg2a cells (E14). GAPDH was used as an internal control. (B) Western blot analysis of $\mathrm{BCl} 3$ in $\mathrm{E} 14$ under the leukemia inhibitor factor (LIF) depletion conditions. After seeding, the cells were cultured for 3 days, and then were transferred in LIF depletion media. The samples were extracted at the indicated times. $\beta$-actin was used as an internal control. (C) Quantitative RT-PCR assay of $\mathrm{BCl} 3$ in ESC-derived embryoid bodies. Embryoid bodies were formed by hanging drop culture methods and extracted at the indicated times. Data are normalized to $\beta$-actin and shown relative to E14 cells. $* * * \mathrm{P}<$ 0.005 vs E14 cells. Error bars indicate the mean \pm SEM $(n=3)$. $P$ values were calculated by using one-way ANOVA. (D) Western blot analysis of Bcl3, Nanog, Sox2, and Oct4 in ESC-derived embryoid bodies. $\beta$-actin was used as an internal control.
mESCs, levels of the Bcl3 transcript (Fig. 1C) and protein (Fig. 1D) were decreased in $\mathrm{mESC}$-derived $\mathrm{EBs}$ on days 1-3. Likewise, core regulatory factors, such as Nanog, Oct4, and Sox2, were significantly decreased in mESC-derived EBs compared with E14. These results supported our hypothesis that $\mathrm{BCl} 3$ is important for self-renewal or differentiation of mESCs.

\section{Insufficiency of Bcl3 attenuates pluripotency of mouse embryonic stem cells}

To investigate whether $\mathrm{Bcl} 3$ regulates the pluripotency of $\mathrm{mESC}$, we generated a knockdown of $\mathrm{Bcl} 3$ in $\mathrm{E} 14$ using shRNA. Western blot analysis showed a lower level of $\mathrm{Bcl} 3$ protein in E14 infected with Bcl3 shRNA (shBcl3) than control mESC (shMock) (Fig. 2A). The proliferation ability of shBcl3 was determined through examination of proliferation of
A
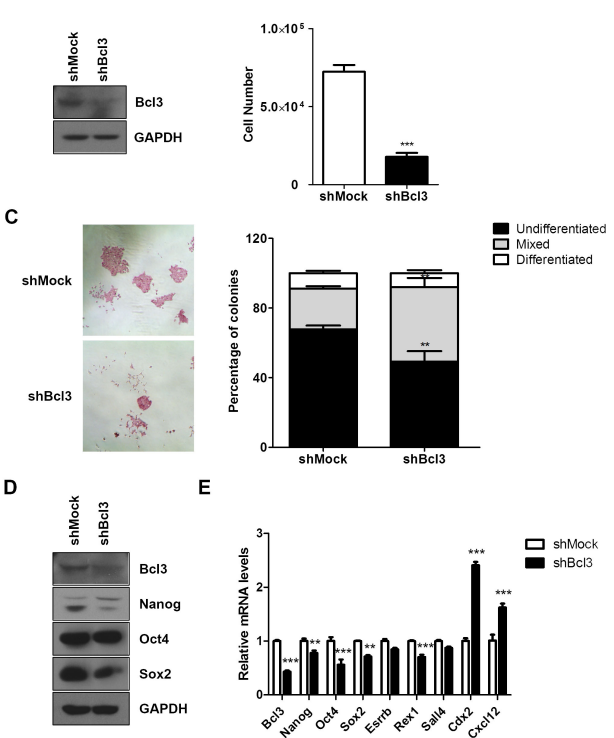

Fig. 2. Knockdown of $\mathrm{Bcl} 3$ attenuated pluripotency of mouse embryonic stem cells. (A) Western blot analysis of Bcl3 in E14 cells transfected with mock vector (shMock) and Bcl3 shRNA (shBcl3). GAPDH was used as an internal control. (B) Cell proliferation assay of shMock and shBcl3. The cells were seeded on a 24-well culture plate at a density of $3 \times 10^{3}$ cells/well and cultured for 3 days. $* * * \mathrm{P}<$ 0.005 vs shMock. (C) Alkaline phosphatase staining of shMock and shBcl3. After AP staining, the colonies were scored, and the percentages of undifferentiated, mixed, and differentiated colonies were calculated. The bar graph shows the statistical evaluation. Error bars indicate the mean \pm SEM $(n=3)$. $P$ values were calculated by using two-way ANOVA. $* * P<0.01$ vs shMock. (D) Western blot analysis of pluripotent-related genes, Nanog, Oct4, and Sox2 in shMock and shBcl3. GAPDH was used as an internal control. (E) Quantitative RT-PCR of pluripotent related genes in shMock and shBcl3. The data are normalized to $\beta$-actin and expressed relative to shMock. The error bars indicate the mean \pm SEM $(n=3)$. P values were calculated by using two-way ANOVA. $* * P<0.01, * * * P<$ 0.005 vs shMock. 
low-density mESCs in a 24-well culture plate. After 3 days, the number of trypsinized shBcl3 showed a remarkable 4-fold decrease compared with that of shMock (Fig. 2B). Alkaline phosphatase (AP) staining, a marker of undifferentiated ESCs, showed that the colonies of undifferentiated mESCs were typically packed and stained a deep reddish color. We confirmed that shBcl3 formed fewer completely undifferentiated AP-positive colonies compared with shMock (Fig. 2C). Moreover, the proportions of differentiated colonies and mixed differentiating colonies in $\mathrm{shBcl} 3$ were higher than that in shMock.

In the evaluation of whether the expression of pluripotency genes was changed in mESCs by the knockdown of $\mathrm{Bcl} 3$, western blot analysis revealed reduced expression of pluripotency genes, Nanog, Oct4, and Sox2 in shBcl3. At the transcriptional level, as assessed by qRT-PCR, shBcl3 not only suppressed pluripotency genes but also, significantly increased differentiation genes, including ectodermal marker $\mathrm{CxCl} 12$ and trophectodermal marker $\mathrm{Cdx2}$ (Fig. 2D). Our results indicated the requirement of $\mathrm{Bcl} 3$ for maintenance of $\mathrm{mESC}$ pluripotency.

\section{Excessive Bcl3 expression also attenuated pluripotency of mouse embryonic stem cells}

To investigate whether $\mathrm{Bcl} 3$ was required for maintenance of $\mathrm{mESC}$ pluripotency, we generated the stable expression of $\mathrm{BCl} 3$ in $\mathrm{E} 14$ by using a lentivirus. Western blot analysis revealed a marked overexpression in the level of $\mathrm{Bcl} 3$ protein in E14 infected with Bcl3 (ZsBcl3) compared with E14 infected with mock vector (ZsMock) (Fig. 3A). First, we evaluated that $\mathrm{BCl} 3$ was involved in the self-renewal and proliferation of $\mathrm{mESCs}$. The proliferation ability of $\mathrm{ZsBcl} 3$ was 5-fold lower than that of ZsMock (Fig. 3B), additionally, ZsBcl3 formed fewer completely undifferentiated colonies, and the proportion of mixed differentiating colonies was significantly increased compared with ZsMock (Fig. 3C).

\section{Bcl3 regulates transcription of Nanog by downregulating promoter activity}

$\mathrm{Bcl} 3$ has been reported to act as a transcriptional regulator of genes associated with immune homeostasis, cellular proliferation, and survival $(18,19)$. We established the hypothesis that $\mathrm{Bcl} 3$ acts as a transcriptional regulator of pluripotent related genes in mESCs. Western blot analysis revealed that Nanog expression was markedly decreased in $\mathrm{ZsBcl} 3$. Moreover, other pluripotent factors were slightly affected in ZsBcl3 (Fig. 3D). Similarly, qRT-PCR assay showed that Bcl3 overexpression decreased expression of the Nanog transcript. In ZsBcl3, Nanog, Sox2, Esrrb and Rex1 transcript levels were decreased in comparison with ZsMock and differentiation genes were induced. To evaluate whether the reduction of Nanog expression in $\mathrm{ZsBcl} 3$ was regulated by $\mathrm{Bcl} 3$, we studied whether $\mathrm{BCl} 3$ regulates the promoter activity of Nanog by using a luciferase reporter assay. E14 was co-transfected with Nanog-5p plasmid, including $2.5 \mathrm{~kb}$ before the proximal

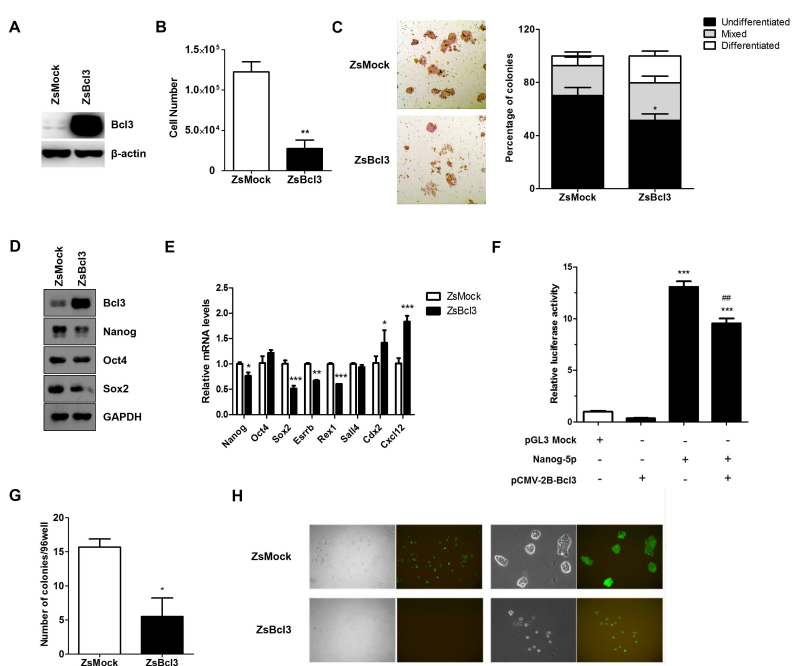

Fig. 3. Overexpression of $\mathrm{Bcl} 3$ reduces pluripotency of mouse embryonic stem cells. (A) Western blot analysis of Bcl3 in E14 cells transfected with mock vector (ZsMock) and Bcl3 (ZsBcl3). $\beta$-Actin was used as an internal control. (B) The cell proliferation assay of ZsMock and $\mathrm{ZsBcl} 3$. ${ }^{*} * \mathrm{P}<0.01$ vs ZsMock. (C) Alkaline phosphatase staining of ZsMock and ZsBcl3. The bar graph shows the statistical evaluation. Error bars indicate the mean \pm SEM $(n=3)$. $P$ values were calculated by using two-way ANOVA. ${ }^{*} \mathrm{P}<0.05$ vs ZsMock. (D) Western blot analysis and (E) quantitative RT-PCR of pluripotent related genes and differentiation related genes in ZsMock and $\mathrm{ZsBcl} 3$. The data are normalized to $\beta$-actin and expressed relative to ZsMock. Error bars indicate the mean \pm SEM $(n=3)$. $P$ values were calculated by using two-way ANOVA. ${ }^{*} \mathrm{P}<0.05,{ }^{*} \mathrm{P}<0.01,{ }^{*} * \mathrm{P}<0.005$ vs ZsMock. (F) Luciferase reporter assay of Nanog in E14 cells. E14 was co-transfected with Nanog-5p and vectors as indicated. The transfected cells were cultured for $24 \mathrm{~h}$ and the luciferase activity was measured. Data were normalized to a Renilla luciferase control. The error bars indicate the mean \pm SEM $(n=4)$. P values were calculated by using one-way ANOVA. ${ }^{* * * P}<0.005$ vs control, ${ }^{\# \#} \mathrm{P}<0.01$ vs Nanog-5p-only transfected cells. (G) Single cells of ZsMock and ZsBcl3 were sorted into 96-well plates by FACS and cultured for 5 days, after which the wells were scored for the presence of colonies. ${ }^{*} \mathrm{P}<0.05$ vs. ZsMock. Error bars indicate the mean \pm SEM $(n=3)$. (H) The morphology of E14_ZsMock and E14_ZsBcl3. The cells were grown for 5 days and sorted for GFP-positive cells by FACS. Representative fluorescence microscopy images at $50 \times$ (left) and $200 \times$ (right) magnification are shown.

promoter of Nanog gene, and the $\mathrm{Bcl} 3$ overexpression plasmid. The results showed a significant decrease in the activity of the Nanog promoter in Bcl3-overexpressing E14. Based on these data, we concluded that $\mathrm{Bcl} 3$ downregulated Nanog expression through reduction of Nanog promoter activity in $\mathrm{mESCs}$.

\section{Excessive Bcl3 expression reduces clonogenic potential in mouse embryonic stem cell}

To study the clonogenicity of $\mathrm{ZsBcl} 3$, we performed a single cell-repopulating assay. After single cells were sorted into a 96-well plate by flow cytometry, we examined the proportion 
of undifferentiated GFP-positive colonies over 5 days. Our results revealed that $\mathrm{ZsBcl} 3$ showed markedly less clonogenic potential than ZsMock (Fig. 3F). We confirmed that ZsBcl3 resulted in more differentiation-like cells and fewer colonies. Also, ZsMock displayed a typical compact mESC colony morphology; in contrast, ZsBcl3 exhibited loosely attached cell morphology (Fig. 3G). These results provided supporting evidence for the hypothesis that abnormally expressed Bcl3 attenuate mESCs pluripotency and induce differentiation of mESCs.

\section{DISCUSSION}

ESCs can undergo self-renewal and differentiation into multi-lineage cells. Pluripotency of ESCs is maintained by a core regulatory network, which includes Oct4, Sox2, and Nanog (2). Expression levels of the core regulatory network control are interrelated, and this extended control of expression facilitates ESC maintenance (20). However, the precise regulatory mechanism for the regulation of the core regulatory network machinery is largely unclear. Here, we propose a novel protein, B cell leukemia/lymphoma 3 (Bcl3), which might control the adequacy of pluripotency and self-renewal potential of ESCs.

Accumulated data indicate that $\mathrm{Bcl} 3$ can interact with other transcriptional regulators, including the AP-1 transcription factors, c-Jun and c-fos (14), STAT1 (21), and PPAR $\gamma$ (22). Studies have also reported $\mathrm{BCl} 3$ expression in different types of hematopoietic and solid tumors, yet its function in ESCs have not been investigated. In this report, we demonstrated that $\mathrm{Bcl} 3$ was involved in proliferation and self-renewal of mESCs via the regulation of Nanog expression. Nanog plays an essential role in the control of the pluripotency of ESCs, as well as in early embryonic development, through its activity as a master transcription factor of the core regulatory factors for pluripotency of mESCs. Notably, Nanog expression is restricted to pluripotent cells and Nanog downregulation causes loss of the ability for self-renewal and an acceleration of ESC differentiation $(3,4,6)$. However, little is known about how Nanog expression is regulated. Here, we found that a novel factor, $\mathrm{Bcl} 3$, acts as a negative regulator of Nanog expression in $\mathrm{mESCs}$. We found that the ectopic expression of $\mathrm{BCl} 3$ decreased Nanog expression. To determine whether the downregulation of Nanog was a result of direct or indirect binding of $\mathrm{BCl} 3$ to the Nanog promoter region, a luciferase reporter assay was performed. The results indicated that Nanog reporter activity was decreased when exposed to overexpressed $\mathrm{Bcl} 3$. Previous reports demonstrated that $\mathrm{Bcl} 3$ regulated Oct4 promoter activity and pluripotency genes in $\mathrm{mESCs}$, and that the expression of Oct4 increased the expression of doxycycline-inducible $\mathrm{Bcl} 3$ in $\mathrm{mESCs}$ (17). However, there was no significant change in the Oct4 expression in our system. One explanation for this discrepancy was the different expression systems. In previous reports, ectopically induced expression of $\mathrm{Bcl} 3$ was increased 1.42-fold, which partially prevented cell differentiation and promoted Oct4 promoter activities. However, the ectopic expression of $\mathrm{BCl} 3$ was $5.7-$ fold higher compared with control mESC, which eventually attenuated the pluripotency and promoted the differentiation of mESCs. Therefore, it is interesting that an excessive dose of $\mathrm{BCl} 3$ negatively impacts mESC pluripotency. Collectively, we concluded that an adequate concentration of B cell leukemia/lymphoma 3 ( $\mathrm{Bcl} 3$ ) is required for the pluripotency and self-renewal of mouse embryonic stem cells ( $\mathrm{mESCs}$ ).

Although we found that $\mathrm{Bcl} 3$ might influence Nanog promoter activity in $\mathrm{mESCs}$, the direct and indirect molecular mechanisms should be further investigated. Previously reported data clearly showed that $\mathrm{Bcl} 3$ repressed TORC3transcription through recruitment of HDAC1 (16), as well as recruitment of HDAC-3 and -6 through association with $\mathrm{Bcl} 3$, to promoter repress transcriptional activity (23). Based on these previous reports, Bcl3 may downregulate Nanog promoter activity through recruitment of the HDAC family or other transcriptional repressors to promoters of Nanog, but this requires further investigation.

A previous study reported that p53 induced the differentiation of DNA-damaged ESCs through the direct suppression of Nanog expression (24). Downregulation of Nanog mRNA during ESC differentiation correlated with the induction of p53 transcriptional activity, which was confirmed by the p53 protein levels in Bcl3-overexpressing mESCs. The results showed that levels of the p53 protein was markedly increased in ZsBcl3 compared with ZsMock (Supplementary Fig. 1). Based on these results, we suggested that $\mathrm{Bcl} 3$ downregulated Nanog expression through p53 induction in mESCs.

In conclusion, $\mathrm{BCl} 3$ is highly expressed in $\mathrm{mESCs}$ and the knockdown of the $\mathrm{Bcl} 3$ gene attenuates the pluripotency of mESCs. Similarly, Bcl3 overexpression also attenuated the pluripotency of $\mathrm{mESCs}$ and promoted the differentiation of mESC through the downregulation of Nanog expression, which suggested that an adequate concentration of $\mathrm{Bcl} 3$ in $\mathrm{mESCs}$ performs a critical role in the maintenance of self-renewal of $\mathrm{mESCs}$ via regulation of Nanog transcriptional activity (Fig. 4).

\section{MATERIALS AND METHODS}

\section{Cell culture and differentiation}

Mouse ES cells (E14tg2a line) were purchased from ATCC (Manassas, VA). mESCs were cultured in Dulbecco's Modified Eagle's Medium (Welgene) containing 15\% fetal bovine serum (Gibco), $1 \times$ non-essential amino acids (Welgene), $0.1 \mathrm{mM}$ $\beta$-mercaptoethanol, and $1,000 \mathrm{U} / \mathrm{ml}$ leukemia inhibitory factor (LIF) (ESGRO, Millipore) on $0.1 \%$ gelatin-coated dishes. Embryoid body formation was performed in hanging drop culture at a concentration of 800 cells per $20 \mu \mathrm{l}$ drop, without LIF. 


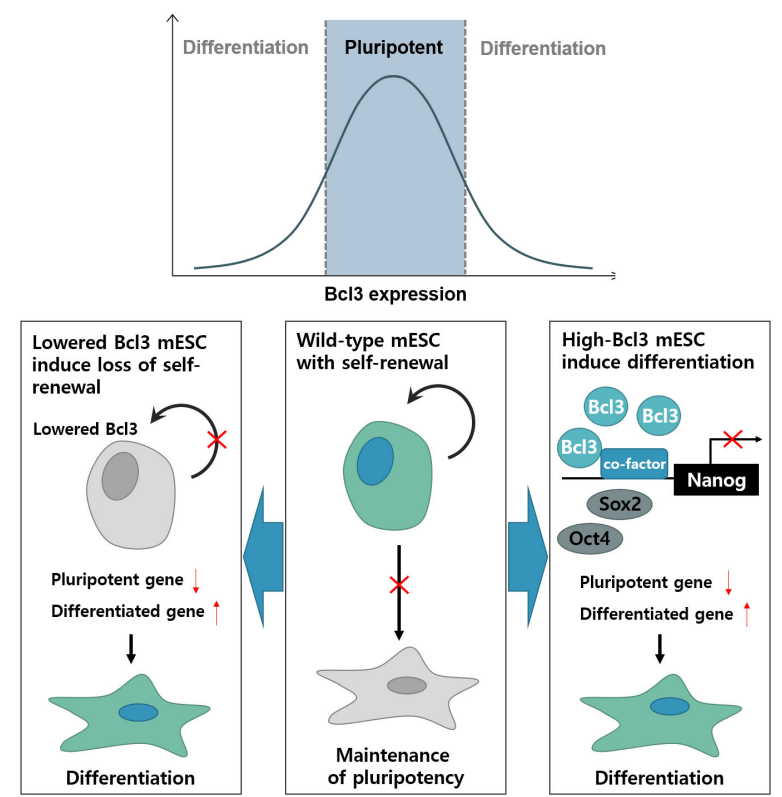

Fig. 4. Regulation of $\mathrm{Bcl} 3$ expression is an essential for maintenance of mouse embryonic stem cell pluripotency. In the absence or overexpression of $\mathrm{Bcl} 3$, mouse embryonic stem cells lose pluripotency and differentiation is promoted. Reduction of Bcl3 in mESCs induces loss of self-renewal ability and induces differentiation. Overexpression of $\mathrm{Bcl} 3$ in mESCs also promotes differentiation through a reduction in Nanog promoter activity. After the suppression of Nanog promoter activity, the expression of pluripotency genes was decreased, and that of differentiation genes, was increased. Collectively, $\mathrm{Bcl} 3$ was required for pluripotency of mESCs and the regulation of $\mathrm{Bcl} 3$ expression is essential for the maintenance of mESC pluripotency.

\section{Lentivirus production and induction of ESC}

For gene overexpression, $\mathrm{Bcl} 3$ was cloned into the pLVX-EF1 $\alpha$-IRES-ZsGreen1 vector. LVX-293T cells were transfected using $2.5 \mu \mathrm{g}$ pMD2G, $7.5 \mu \mathrm{g}$ psPAX, $10 \mu \mathrm{g}$ plasmid, and $30 \mu \mathrm{l}$ Lipofectamine 2000 (ThermoFisher Scientific). For short hairpin RNA (shRNA) knockdowns, Bcl3 shRNA constructs were purchased from Sigma. Viral particles were collected $48 \mathrm{~h}$ after transfection and filtered through a $0.45 \mu \mathrm{m}$ filter (Sartorius). Mouse ES cells were infected by replacement of the culture medium with $5 \mathrm{ml}$ of viral supernatant and $5 \mathrm{ml}$ complete medium in the presence of 5 $\mu \mathrm{g} / \mathrm{ml}$ polybrene (Sigma) and incubated for $16 \mathrm{~h}$. Overexpressed mESCs were sorted for GFP expression using fluorescence-activated cell sorting (FACS). For knockdown experiments, the expression of shRNA was selected by addition of $5 \mu \mathrm{g} / \mathrm{ml}$ puromycin.

\section{Single cell repopulation assay}

pLVX-EF1 $\alpha$-IRES-ZsGreen1-Bcl3 transduced E14 cells were sorted for GFP expression using flow cytometry (BD FACS Aria I). Flow cytometry was used to place a single GFP-positive E14 in the well of a 96-well culture plate. Each well was coated with $0.1 \%$ gelatin and contained $200 \mu \mathrm{l}$ complete medium. The medium was replaced with fresh, complete medium every 2 days. After 5 days, each well was examined for the growth of mouse ES cells by fluorescence microscopy (Zeiss). To measure the number of cells in a colony, we visually counted the cells in each of the 96 wells by using an inverted phase-contrast microscope at $\times 40$ magnification.

\section{Alkaline phosphatase staining}

mESCs were seeded on a $0.1 \%$ gelatin coated 24-well culture plate at a density of $3 \times 10^{3}$ cells/well and cultured for 3 days. Alkaline phosphatase staining was performed using the Alkaline Phosphatase Kit (Millipore) in accordance with the manufacturer's instructions.

\section{RNA isolation and quantitative RT-PCR}

Total RNA was isolated by using TRlzol Reagent (ThermoFisher Scientific) and cDNA was synthesized with PrimeScript 1st Strand cDNA Synthesis Kit (Takara) using oligo(dT) primer. Quantitative RT-PCR was performed on a LightCycler 96 Real-Time PCR System (Roche) using FastStart Essential DNA Green Master (Roche). The relative expression of each gene was normalized to $\beta$-actin expression. The primer sequences used are listed in Supplemental Table 1.

\section{Westem blot analysis}

Total protein was extracted by using RIPA lysis buffer (ThermoFisher Scientific). Cell lysates were separated by SDS-PAGE and transferred onto a PVDF (Millipore). PVDF was probed with primary antibodies against Nanog (Bethyl), Bcl3, Sox2, Oct4, p53, GAPDH, $\beta$-actin (Santacruz) followed by the application of HRP-conjugated secondary antibodies. HRP was detection by the application of $\mathrm{ECL}$ reagent (Millipore) to PVDF and exposure to x-ray films.

\section{Luciferase reporter assay}

E14tg2a cells were plated in 24-well plates and were transfected with Nanog-5P, which contains $2.5 \mathrm{~kb}$ of the $5^{\prime}$ promoter region of the mouse Nanog gene (Addgene), pCMV-2B-Bcl3 using Lipofectamine 3000 reagent in accordance with the manufacturer's protocol (ThermoFisher Scientific). pCMV-2B-Bcl3 was cloned into the multiple cloning site $(\mathrm{BamHI}$ and $\mathrm{Xhol})$ of the pCMV Tag $2 \mathrm{~B}$ vector (Agilent technologies). The cells were extracted $24 \mathrm{~h}$ after transfection and the extracted cells were analyzed by using the Dual-Luciferase Reporter Assay (Promega), with the luciferase activity measured in a multilabel plate reader (VICTOR3). The relative luciferase activity of each sample was normalized to Renilla activity.

\section{Statistical analysis}

Statistical analysis was performed with a two-tailed unpaired Student's t-test, or one-way or two-way ANOVA. The data 
were reported as the mean \pm standard deviation. A value of $\mathrm{P}<0.05$ was considered statistically significant

\section{ACKNOWLEDGEMENTS}

This work was supported by the National Research Foundation of Korea (NRF) grant funded by the Korea government (MSIP) (NRF-2015M3A9B4066493, NRF-2015M3A9B4051053, NRF2015R1A5A2009656, NRF-2014R1A2A1A11052311).

\section{CONFLICTS OF INTEREST}

The authors have no conflicting interests.

\section{REFERENCES}

1. Suda Y, Suzuki M, Ikawa Y and Aizawa S (1987) Mouse embryonic stem cells exhibit indefinite proliferative potential. J Cell Physiol 133, 197-201

2. Niwa H (2007) How is pluripotency determined and maintained? Development 134, 635-646

3. Chambers I, Colby D, Robertson M et al (2003) Functional expression cloning of Nanog, a pluripotency sustaining factor in embryonic stem cells. Cell 113, 643-655

4. Mitsui K, Tokuzawa Y, Itoh H et al (2003) The homeoprotein Nanog is required for maintenance of pluripotency in mouse epiblast and ES cells. Cell 113, 631-642

5. Boyer LA, Lee TI, Cole MF et al (2005) Core transcriptional regulatory circuitry in human embryonic stem cells. Cell 122, 947-956

6. Chambers I, Silva J, Colby D et al (2007) Nanog safeguards pluripotency and mediates germline development. Nature 450, 1230-1234

7. Kuroda T, Tada M, Kubota $\mathrm{H}$ et al (2005) Octamer and Sox elements are required for transcriptional cis regulation of Nanog gene expression. Mol Cell Biol 25, 2475-2485

8. Rodda DJ, Chew JL, Lim LH et al (2005) Transcriptional regulation of nanog by OCT4 and SOX2. J Biol Chem 280, 24731-24737

9. van den Berg DL, Zhang W, Yates A et al (2008) Estrogen-related receptor beta interacts with Oct4 to positively regulate Nanog gene expression. Mol Cell Biol 28, 5986-5995

10. Ohno H, Takimoto G and McKeithan TW (1990) The candidate proto-oncogene bcl-3 is related to genes implicated in cell lineage determination and cell cycle control. Cell 60, 991-997

11. Maldonado V and Melendez-Zajgla J (2011) Role of Bcl-3 in solid tumors. Mol Cancer 10, 152

12. Fujita T, Nolan GP, Liou HC, Scott ML and Baltimore D (1993) The candidate proto-oncogene bcl-3 encodes a transcriptional coactivator that activates through NF-kappa B p50 homodimers. Genes Dev 7, 1354-1363

13. Inoue J, Takahara T, Akizawa T and Hino O (1993) Bcl-3, a member of the I kappa B proteins, has distinct specificity towards the Rel family of proteins. Oncogene 8, 2067-2073

14. Na S-Y, Choi J-E, Kim H-J, Jhun BH, Lee Y-C and Lee JW (1999) $\mathrm{BCl} 3$, an IKB protein, stimulates activating protein-1 transactivation and cellular proliferation. J Biol Chem 274, 28491-28496

15. Na S-Y, Choi H-S, Kim JW, Na DS and Lee JW (1998) $\mathrm{BCl} 3$, an I $\mathrm{KB}$ protein, as a novel transcription coactivator of the retinoid X receptor. J Biol Chem 273, 30933-30938

16. Hishiki T, Ohshima T, Ego T and Shimotohno K (2007) $\mathrm{BCL} 3$ acts as a negative regulator of transcription from the human T-cell leukemia virus type 1 long terminal repeat through interactions with TORC3. J Biol Chem 282, 28335-28343

17. Chen CY, Lee DS, Yan YT et al (2015) Bcl3 Bridges LIF-STAT3 to Oct4 Signaling in the Maintenance of Naive Pluripotency. Stem Cells 33, 3468-3480

18. Bours V, Franzoso G, Azarenko $V$ et al (1993) The oncoprotein Bcl-3 directly transactivates through $\mathrm{\kappa B}$ motifs via association with DNA-binding p50B homodimers. Cell 72, 729-739

19. Park SG, Chung C, Kang H, Kim J-Y and Jung G (2006) Up-regulation of cyclin $\mathrm{D} 1$ by $\mathrm{HBx}$ is mediated by NF- $\kappa B 2 / B C L 3$ complex through $\kappa B$ site of cyclin D1 promoter. J Biol Chem 281, 31770-31777

20. Loh YH, Wu Q, Chew JL et al (2006) The Oct4 and Nanog transcription network regulates pluripotency in mouse embryonic stem cells. Nat Genet 38, 431-440

21. Jamaluddin $M$, Choudhary $S$, Wang $S$ et al (2005) Respiratory syncytial virus-inducible BCL-3 expression antagonizes the STAT/IRF and NF- $\mathrm{KB}$ signaling pathways by inducing histone deacetylase 1 recruitment to the interleukin-8 promoter. J Virol 79, 15302-15313

22. Yang J, Williams RS and Kelly DP (2009) Bcl3 interacts cooperatively with peroxisome proliferator-activated receptor gamma (PPAR $\gamma$ ) coactivator $1 \alpha$ to coactivate nuclear receptors estrogen-related receptor $\alpha$ and PPAR $\alpha$. Mol Cell Biol 29, 4091-4102

23. Viatour P, Dejardin E, Warnier M et al (2004) GSK3-mediated BCL-3 phosphorylation modulates its degradation and its oncogenicity. Mol Cell 16, 35-45

24. Lin T, Chao C, Saito S et al (2005) p53 induces differentiation of mouse embryonic stem cells by suppressing Nanog expression. Nat Cell Biol 7, 165-171 